\title{
Avaliação da Usabilidade do Scratch utilizando o Método System Usability Scale (SUS)
}

\author{
Wendell Bento Geraldes, Ernane Rosa Martins, Ulisses Rodrigues Afonseca ${ }^{1}$
}

${ }^{1}$ Núcleo de Inovação, Tecnologia e Educação - Instituto Federal de Goiás campus Luziânia 72.812-580 - Luziânia - GO - Brasil

\{wendell.geraldes, ernane.rosa, ulisses.afonseca\}@ifg.edu.br

\begin{abstract}
The Scratch visual programming language was created in 2007 at MIT, making it widely used worldwide. This article aims to evaluate the usability of the language in its on-line version available on the internet. For this purpose we used the System Usability Scale (SUS) method, created by John Brooke in 1986, which is considered one of the most reliable and valid questionnaires to measure the perceived usability of users. Data collection was performed through an on-line questionnaire containing 10 questions, with a scale from 1 to 5, where 1 means Strongly Disagree and 5 Strongly Agree. The results showed a positive evaluation of users regarding the Scratch language.
\end{abstract}

Resumo. A linguagem visual de programação Scratch foi criada em 2007 no MIT, tornando-se largamente utilizada no mundo inteiro. Este artigo tem como objetivo avaliar a usabilidade da linguagem em sua versão on-line disponível na internet. Para esta finalidade foi utilizado o método System Usability Scale (SUS), criado por John Brooke em 1986, que é considerado um dos questionários mais confiáveis e válidos para medir a usabilidade percebida pelos usuários. A coleta de dados foi realizada através de um questionário on- line contendo 10 perguntas, com uma escala de 1 a 5, onde 1 significa Discordo totalmente e 5 Concordo totalmente. Os resultados obtidos demostraram uma avaliação positiva dos usuários em relação a linguagem Scratch.

\section{Introdução}

O Scratch é uma linguagem de programação visual desenvolvida por uma equipe do Massachusetts Institute of Technology - MIT e lançada em 2007, possui uma comunidade de usuários espalhada por todo o mundo e conta coma mais de 42 milhões de projetos compartilhados. Atualmente estão registrados mais de 41 milhões de usuários, sendo que somente no Brasil são cerca de 506 mil [Scratch 2019]. A linguagem está disponível por meio de uma plataforma Web, podendo também ser instalada em computadores pessoais que não possuem acesso a internet. O Scratch permite a criação de jogos, animações e histórias interativas digitais. Estes projetos podem ser compartilhados e modificados por outros usuários da plataforma [Eloy et al. 2017].

O uso do Scratch pode possibilitar aos estudantes a criação de seus próprios conteúdos relacionados a sua área curricular, com inúmeras vantagens, tais como: motivação, diversão, comprometimento e entusiasmo, além de apresentar melhorias relacionadas ao pensamento computacional e as práticas computacionais [Sáez-López et al. 2016]. A linguagem possui uma interface simples e muito intuitiva, 
sendo baseada no uso de blocos que são peças usadas para criar os códigos. Os blocos conectam-se uns aos outros como peças de um quebra-cabeça, onde cada tipo de dado (evento, comando, valor reportado, valor booleano, ou fim do script) tem sua própria forma específica que se encaixam perfeitamente uns aos outros. Uma série de blocos conectados são chamados de Scripts [Scratch 2019]. Apesar de ser bastante conhecida, não existem muitas pesquisas sobre sua usabilidade.

Este trabalho tem como objetivo avaliar a usabilidade da linguagem Scratch, utilizando o método System Usability Scale (SUS), que é uma métrica de avaliação da usabilidade de sistemas computacionais, constituído por um questionário padronizado, considerado o mais utilizado do mundo por sua praticidade e rapidez. Quando os pesquisadores e profissionais precisam de uma medida de usabilidade, estes certamente consideram seriamente o uso do SUS [Lewis 2018]. Este é considerado um dos questionários mais confiáveis e válidos para medir a usabilidade percebida pelos usuários, mesmo com a utilização de pequenas amostras [Revythi and Tselios 2019].

\section{Usabilidade}

Segundo [Nielsen 2006], usabilidade é um atributo de qualidade que avalia a facilidade de uso de uma interface, sendo definida por cinco componentes:

1. Capacidade de aprendizagem: a facilidade de utilizar o sistema pela primeira vez;

2. Eficiência: rapidez para executar as tarefas;

3. Memorização: o processo de lembrar como utilizar o sistema, após um tempo sem utilizar;

4. Erros: ausência de erros apresentados pelo sistema;

5. Satisfação: design agradável.

[Shackel 1986] sugere quatro critérios para a avaliação das tarefas sob o ponto de vista da usabilidade:

1. Eficácia: avalia o desempenho de uma tarefa tendo como foco a análise da velocidade de execução e quantidade de erros;

2. Aprendizagem: avalia o desempenho desde a instalação do produto até o início do uso;

3. Flexibilidade: Avalia a capacidade de adaptação a novas tarefas além das já especificadas no sistema;

4. Atitude: Avalia o desempenho com relação ao conforto ou satisfação do usuário ao utilizar o sistema.

[Bastien and Scapin 1993] apresentaram oito critérios ergonômicos para construção de interfaces de sistemas:

1. Condução: refere-se aos meios disponíveis para aconselhar, orientar, informar, e conduzir o usuário na interação com o computador (mensagens, alarmes, rótulos, etc.).

2. Carga de Trabalho: está relacionada aos elementos da interface, devido ao importante papel na redução da carga cognitiva e perceptiva do usuário, possibilitando aumento da eficiência do diálogo.

3. Controle Explícito: refere-se ao controle que os usuários tem sobre o processamento de suas ações pelo sistema. 
4. Adaptabilidade: capacidade do software em se comportar conforme as necessidades e preferências do usuário.

5. Gestão de erros: compreende os mecanismos capazes de prevenir e reduzir erros causados pelo sistema, além de rotinas para facilitar sua correção quando os mesmos ocorrerem.

6. Consistência: o modo que os aspectos de interface são mantidos em contextos semelhantes, e se diferenciam em contextos distintos.

7. Significância do código: relação semântica entre os códigos e os itens ou ações às que eles se referem, demonstra a clareza com que os códigos e denominações são apresentados para o usuário.

8. Compatibilidade: é a relação entre as características dos usuários e as tarefas, saídas e entradas do sistema.

\section{Técnicas de Avaliação de Usabilidade}

Para [Cybis 2003] existem três tipos de técnicas de avaliação ergonômica (Figura 1):

1. Técnicas prospectivas, que buscam a opinião do usuário sobre a interação com o sistema;

2. Técnicas preditivas ou diagnósticas, que buscam prever os erros de projeto de interfaces sem a participação de usuários;

3. Técnicas objetivas ou empíricas, que buscam constatar os problemas a partir da obervação do usuário interagindo com o sistema.

Figura 1. Técnicas de avaliação de usabilidade

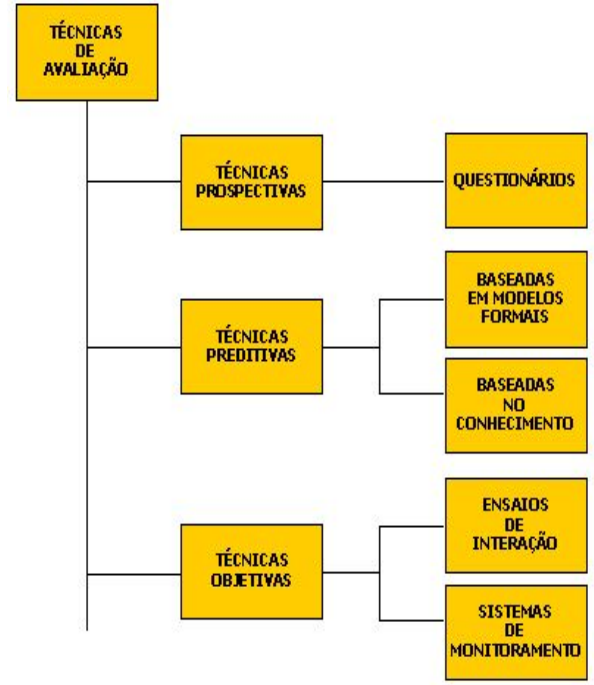

Fonte: [Cybis 2003]

As técnicas prospectivas estão baseadas na aplicação de questionários/entrevistas com o usuário para avaliar sua satisfação ou insatisfação em relação ao sistema e sua operação. Ela mostra-se bastante pertinente na medida em que é, o usuário a pessoa que melhor conhece o software. Nada mais natural em buscar suas opiniões para orientar revisões de projeto. Este tipo de técnica pode ser empregado para aumentar a efetividade de avaliações analíticas, realizadas por especialistas que diagnosticam problemas de usabilidade. Apoiados pelas respostas do questionário de satisfação, estes podem centrar suas análises sobre os pontos problemáticos no sistema, apontados pelo usuário [Cybis 2003]. 


\section{System Usability Scale (SUS)}

Existem vários questionários disponíveis para profissionais de usabilidade que podem auxiliar a avaliação da usabilidade de um produto ou serviço. Muitos destes questionários são usados para avaliar tipos específicos de interfaces, enquanto outros podem ser usados para avaliar uma faixa mais ampla de tipos de interface. A escala System Usability Scale (SUS) é uma das que podem ser usadas para avaliar a usabilidade de uma variadade de produtos ou serviços. As características do SUS que tornam seu uso atrativo, são:

- Composto de apenas dez afirmações: é relativamente rápido e fácil para os participantes do estudo concluírem e para os administradores pontuarem;

- Não proprietário: rentável para uso e pode ser pontuado muito rapidamente, imediatamente após a conclusão;

- Independente de tecnologia: pode ser usado por um amplo grupo de profissionais de usabilidade para avaliar praticamente qualquer tipo de interface de usuário;

- Pontuação única: variando de 0 a 100 , sendo relativamente fácil de entender por uma ampla gama de pessoas de outras disciplinas que trabalham em equipes de projeto [Bangor et al. 2009].

No estudo realizado por [Bangor et al. 2009] os resultados de 2.324 pesquisas do SUS em 206 testes de usabilidade coletados ao longo de um período de dez anos, verificou-se que o SUS era altamente confiável $($ alfa $=0,91)$ e útil em uma ampla gama de tipos de interface [Bangor et al. 2009].

O conceito de aplicar um adjetivo (bom, ruim, excelente, etc.) à usabilidade do produto era atraente porque é familiar para a maioria das pessoas que trabalham em equipes de design, independentemente de sua disciplina. Ter um ponto de referência familiar e fácil de entender que pode ser facilmente entendido por engenheiros e gerentes de projeto facilita a comunicação dos resultados do teste. [Bangor et al. 2009] relataram os resultados de um estudo piloto que procurou mapear adjetivos descritivos (por exemplo, bom, péssimo, etc.) ao alcance das pontuações do SUS [Bangor et al. 2009].

\section{Metodologia}

Neste estudo foi realizada uma pesquisa exploratória através de um formulário on-line com objetivo de conhecer as opiniões dos usuários acerca da usabilidade da linguagem de programação visual Scratch. Para coleta de dados foi utilizado um questionário SUS - System Usability Scale composto por 10 itens, com 5 opções de respostas. O respondente do questionário SUS assinala sua resposta numa escala Likert que varia de 1 (Discordo totalmente) a 5 (Concordo totalmente) observado na Figura 2 [Boucinha and Tarouco 2013].

Figura 2. Exemplo de questão do SUS

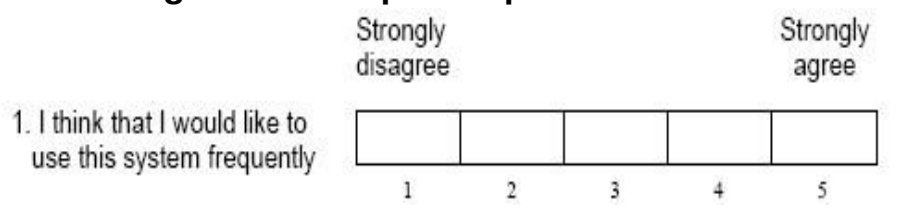

Fonte: [Boucinha and Tarouco 2013]

O questionário SUS foi construído em língua inglesa, porém optou-se por utilizar uma versão traduzida para o português conforme a Tabela 1, por ser mais adequada ao 
público alvo da pesquisa [Teixeira 2015]. A análise dos resultados do SUS foi a soma da contribuição individual de cada item. Para os itens ímpares deve-se subtrair 1 à resposta do usuário, ao passo que para os itens pares deve-se subtrair 5 a resposta do usuário. Depois de obter o score de cada item, soma-se os scores e multiplica-se o resultado por 2,5. O resultado obtido será um índice de satisfação do utilizador que varia de 0 a 100 [Boucinha and Tarouco 2013]. A pesquisa pode ser classificada como descritiva. Para [Gil 2010] uma pesquisa descritiva tem como objetivo a descrição de características de uma determinada população, utilizando técnicas padronizadas de coleta de dados, como questionários e observação sistemática [Gil 2010].

Tabela 1. Questionário System Usability Scale traduzido para o português

\begin{tabular}{|c|l|}
\hline Item & Item correspondente em português \\
\hline 1 & Acho que gostaria de utilizar este produto com frequência \\
\hline 2 & Eu acho o sistema desnecessariamente complexo \\
\hline 3 & Eu achei o sistema fácil de usar. \\
\hline 4 & $\begin{array}{l}\text { Eu acho que precisaria de ajuda de uma pessoa com conhecimentos técnicos para } \\
\text { usar o sistema. }\end{array}$ \\
\hline 5 & Eu acho que as várias funções do sistema estão muito bem integradas. \\
\hline 6 & Eu acho que o sistema apresenta muita inconsistência. \\
\hline 7 & Eu imagino que as pessoas aprenderão como usar esse sistema rapidamente. \\
\hline 8 & Eu achei o sistema atrapalhado de usar. \\
\hline 9 & Eu me senti confiante ao usar o sistema. \\
\hline 10 & Eu precisei aprender várias coisas novas antes de conseguir usar o sistema. \\
\hline
\end{tabular}

\section{Resultados}

A coleta de dados foi realizada entre os dias 13/05/2019 e 11/06/2019, sendo obtidas 83 respostas ao questionário SUS. Esta quantidade de respondentes é $21 \%$ do tamanho esperado da amostra, que era de 384 respostas.

A avaliação da usabilidade realizada pelos usuários da linguagem Scratch por meio do questionário SUS revelou que o média geral do SUS-Score foi de 75,27. Este indicador mostra que a linguagem possui uma boa usabilidade [Teixeira 2015].

\section{Considerações finais}

Este trabalho teve como objetivo avaliar a usabilidade da linguagem Scratch, utilizando o método System Usability Scale (SUS). O resultado encontrado com a aplicação do questionário SUS na avaliação da usabilidade da linguagem Scratch na percepção do usuário, teve como classificação o status de bom, por ter alcançado a pontuação de 75,27 [Bangor et al. 2009]. Permitindo inferir que a linguagem Scratch atende as necessidades de seus usuários quanto a usabilidade.

Embora a aplicação do questionário SUS tenha sido amplamente divulgada nos meios de comunicação digitais, a amostra coletada pode ser considerada pequena diante do número de usuários brasileiros da linguagem Scratch. Sendo fundamental a realização de novos estudos para a ampliação da amostra e confirmação do resultado encontrado, 
permitindo uma melhor análise e discussão deste tema. Podendo ainda ser utilizados outros questionários gratuitos na avaliação, tais como: After Scenario Questionnaire (ASQ) da IBM e Usefulness, Satisfaction and Ease of Use (USE).

\section{Referências}

Bangor, A., Kortum, P., and Miller, J. (2009). Determining what individual sus scores mean: Adding an adjective rating scalejus. JUS - The Journal of Usability Studies, 4(3):113-123.

Bastien, J. C. and Scapin, D. L. (1993). Human factors criteria, principles, and recommandations for hci: methodological and standatdisation issues. INRIA.

Boucinha, R. M. and Tarouco, L. M. R. (2013). Avaliação de ambiente virtual de aprendizagem com o uso do sus - system usability scale. Revista Renote, 11(3):1-10. Novas Tecnologias na Educação.

Cybis, W. (2003). Engenharia de usabilidade: Uma abordagem ergonômica. laboratório de utilizabilidade de informática (labiutil). Disponível em: http://www . labiutil.inf.ufsc.br/hiperdocumento/conteudo.html. Acesso em 11 de agosto de 2019.

Eloy, A., Lopes, R., and Angelo, I. (2017). Uso do scratch no brasil com objetivos educacionais: uma revisão sistemática. Revista Renote, 15(1):1-10. Novas Tecnologias na Educação.

Gil, A. C. (2010). Como elaborar projetos de pesquisa. Atlas, 5 edition.

Lewis, J. (2018). The system usability scale: Past, present, and future. International Journals of Human - Computer Interaction, 34(7):577-590.

Nielsen, J. (2006). Usability 101: Introduction to usability. Disponível em: http: / / www. ingenieriasimple.com/usabilidad/IntroToUsability.pdf. Acesso em 11 de junho de 2019.

Revythi, A. and Tselios, N. (2019). Extension of technology acceptance model by using system usability scale to assess behavioral intention to use e-learning. Education and Information Technologies, 24(4):2341-2355.

Scratch (2019). Estatísticas. Disponível em: <https://scratch.mit.edu/ statistics/>. Acesso em 11 de junho de 2019.

Shackel, B. (1986). Ergonomics in design for usability. In: HCI 86 Conference on People and Computer. New York: Cambridge University Press.

Sáez-López, J., Román-Gonzáles, M., and Vázquez-Cano, E. (2016). Visual programming languages integrated across the curriculum in elementary schools: A two years case study using scratch in five schools. Computer \& Education, 97:129-141.

Teixeira, F. (2015). O que é o sus (system usability scale) e como usá-lo em seu site. Disponível em: <https://brasil.uxdesign.cc/o-queC $3 \backslash \% A 9-0-$ sus-system-usability-scale-e-como-us \\%C3\ A1-lo-em-seu-site-6d63224481c8>. Acesso em 11 de junho de 2019. 